\title{
Epigenetics in Osteoarthritis Related Pain: An Update
}

\author{
Jorge Hugo VILLAFAÑE' ${ }^{10}$, Paolo PEDERSINI ${ }^{1}$, Pedro BERJANO ${ }^{2}$ (i) \\ ${ }^{1}$ Department of Clinical Research, IRCCS Fondazione Don Carlo Gnocchi, Milan, Italy \\ ${ }^{2}$ Department of GSpine4, IRCCS Istituto Ortopedico Galeazzi, Milan, Italy
}

Osteoarthritis (OA) is the most common form of arthritis worldwide and is a major cause of pain and disability in elderly people. ${ }^{1}$ The discrepancies between pain in $\mathrm{OA}$ and severity of radiographic findings are usually associated by the propensity of some patients to show sensitization phenotype and supported by recent theories that OA involves complex mechanisms of altered pain transmission. ${ }^{2,3}$ Pain in $\mathrm{OA}$ is related to innumerable internal and external factors including environmental changes as diet, exercise, disease, stress but also genetic. OA has a strong genetic component but the success of previous studies has been restricted due to insufficient sample sizes and phenotype heterogeneity. The field of epigenetics continues its influential rise as a mean to better understand an organism's unique developmental identity over a lifespan. ${ }^{4}$ Epigenetics is particularly interesting in medical research both for the potential to cause disease and as a target for therapeutic interventions.

Recent studies employed a targeted approach on known genes to play a role in OA pathophysiology; for example, OA responsive deoxyribonucleic acid (DNA) methylation changes seem to mediate disease associated aberrant gene expression. ${ }^{5}$
Furthermore, established OA susceptibility alleles such as GDF5 and DIO2 appear to confer OA risk via DNA methylation and respective pathophysiological expression changes. Aside from rare mutations in genes encoding structural proteins in articular cartilage, few common genes have been identified as risk factors for the development of hip or knee OA in genome-wide association studies. ${ }^{4}$ Recently, genome wide profiling of DNA methylation in OA affected articular cartilage has emerged as a powerful tool to address the epigenetic changes in their entirety. ${ }^{5}$ Epigenetic alterations produced after injury contribute to the generation and maintenance of neuropathic pain involving the central and peripheral nervous system. The current literature describes clear effects of DNA methylation, histone methylation and acetylation, and micro-ribonucleic acids on the expression of ion channels, receptors and neurotransmitters in neurons. Several histone acetyltransferases affect chemokine expression whereas histone deacetylases affect cytokine expression within glial and macrophage cells that are reactive to neuronal damage. However, there is no agreement regarding histone and DNA methylation effects on inflammatory mechanisms that sustain pain states. ${ }^{6}$ Restoring DNA methylation may represent

Received: June 11, 2019 Accepted: October 09, 2019 Published online: January 08, 2020

Correspondence: Jorge Hugo Villafañe, MD. Department of Clinical Research, IRCCS Fondazione Don Carlo Gnocchi, 20121, Milan, Italy. Tel: +393395857563 e-mail: mail@villafane.it 
a new therapeutic approach to treat OA related pain. $^{7}$

Studies on epigenetics and pain remain largely preclinical and investigate the theoretical ability of epigenetics to alter the nociceptive pathways both peripherally and centrally. Significant evidence emerged in the ability of epigenetics to modify broadly categorized pain types, including inflammatory, neuropathic, visceral and cancer related. Tightly regulated epigenetic alterations on DNA and histones in the sensory circuit shape the physiological response to injury. Altering those epigenetic processes hinder therapeutic potential in pain.

Although a rising interest is shown within the epigenetics field, we are still at the preliminary steps of understanding these processes. Prospective studies, evaluating the epigenetics -host relationship, are indeed necessary to establish not only the potential etiology, but also the effects of anti-inflammatory therapies on the $\mathrm{OA}$ and how they may impact on patient's wellbeing.

\section{Declaration of conflicting interests}

The authors declared no conflicts of interest with respect to the authorship and/or publication of this article.

\section{Funding}

The authors received no financial support for the research and/or authorship of this article.

\section{REFERENCES}

1. Villafañe JH. Does "time heal all wounds" still have a future in osteoarthritis? Clin Exp Rheumatol 2018;36:513.

2. Clauw DJ, Hassett AL. The role of centralised pain in osteoarthritis. Clin Exp Rheumatol 2017;35 Suppl 107:79-84.

3. Villafañe $\mathrm{JH}$, Valdes $\mathrm{K}$, Pedersini $\mathrm{P}$, Berjano P. Osteoarthritis: a call for research on central pain mechanism and personalized prevention strategies. Clin Rheumatol 2019;38:583-4.

4. Hochberg MC, Yerges-Armstrong L, Mitchell BD. Osteoarthritis susceptibility genes continue trickling in. Lancet 2012;380:785-7.

5. den Hollander W, Meulenbelt I. DNA Methylation in Osteoarthritis. Curr Genomics 2015;16:419-26.

6. Penas C, Navarro X. Epigenetic Modifications Associated to Neuroinflammation and Neuropathic Pain After Neural Trauma. Front Cell Neurosci 2018;12:158.

7. Garriga J, Laumet G, Chen SR, Zhang Y, Madzo $\mathrm{J}$, Issa JJ, et al. Nerve Injury-Induced Chronic Pain Is Associated with Persistent DNA Methylation Reprogramming in Dorsal Root Ganglion. J Neurosci 2018;38:6090-101. 